\title{
Dealing with garbage is the immune system's main job
}

\begin{abstract}
An alternative view of the immune system as a garbage handling system provides a different framework for explanation of the normal and abnormal responses of the system, including autoimmunity. As such, the normal functions and roles of immune components are to maintain the status quo which consists of processing and recycling molecular and cellular garbage including the response to microbial debris.
\end{abstract}

Keywords: dead cells, exosomes, immune system
Volume 5 Issue 6 - 2017

\author{
Ken S Rosenthal \\ Department of Biomedical Sciences, Roseman University \\ College of Medicine, USA
}

Correspondence: Ken S Rosenthal, Department of Biomedical Sciences, Roseman University College of Medicine, 10530 Discovery Dr, Las Vegas, NV, 89|35, USA,,Tel 702-802-285-5, Email krosenthal@roseman.edu

Received: January 0I, | 970 | Published: July 12, 2017

\section{Mini Review}

The classical analogy for the immune system is of a military response to invasion. This is appropriate for explaining the complexities of the system's response to infectious challenges but does not deal with the normal, routine, status quo functions of the system, as would occur during peacetime. As with the military, understanding the function of the immune response and its components requires an understanding of their responses and roles in maintenance of normal, status quo, everyday situations of the body and their ability to react to sporadic challenges. What follows is not meant to be a detailed or referenced review but more of an opinion to be shared with others besides my own students. Like a city, the body is constantly creating cellular and molecular garbage. Cells die, proteins denature and without a means for disposing and recycling of these materials, they would build up and clog the system. Macrophages are usually thought of as the garbage processors of the body. Macrophages pick up and recycle the dead (apoptotic, necrotic), broken (denatured proteins) and discarded parts (e.g. Exosomes) of our cells, tissues and body. Tissue and bone marrow derived macrophages carry out this boring task every single day without getting excited or angry. Dendritic cells, including Langerhans cells, are garbage pickers, possibly more selective and more reactive to the type of trash that they pick up than the macrophage. Their role will be described later.

Like garbage persons (they are genderless), macrophages take up garbage indiscriminately and the material may or may not be in containers. Dead cells and Exosomes ${ }^{1}$ provide receptacles for garbage but degraded, denatured proteins and particles are strewn across the body's terrain. Dead and denatured material exposes unique structures that distinguish them from viable materials. These structures can be recognized by opsonins, one end of which binds to the molecular or cellular garbage and the other end to a receptor on the macrophage. As such, the opsonins facilitate the cleanup process. Opsonins include pentraxins, collectins, ficolins, complement, and antibodies. These molecules bind the structures on proteins, glycoproteins, carbohydrates, lipids and glycolipids, which may be exposed on native or denatured proteins, other molecules, Exosomes, particles, apoptotic or necrotic dead cells, and also microbes and their components. The importance of proper trash marking is indicated by the consequences of defects in the early components of complement leading to autoimmune diseases, such as lupus. ${ }^{2}$
Like many garbage trucks, the surface of macrophages is decorated with pieces of the collected garbage. During recycling of the garbage, phagocytosed proteins are processed and peptides of 1113 amino acid length are selected to decorate the cell surface in major histocompatibility protein (MHC) II holders. The dendritic cell also picks up garbage but decorates its surface using MHC II holders and also MHC I holders. MHC I molecules acquire their 8-9 amino acid peptides by a process called cross-presentation while they travel to the draining lymph node. Each of these cells display 6 different MHC I and MHC II holders to ensure an extensive but somewhat selective array of peptides indicative of the processed protein garbage.

The MHC II molecule displays its peptide to CD4 T cells and the MHC I-peptide complexes to CD8 T cells. These T cells cannot see peptides unless thrust upon them within MHC molecules. In this way, the macrophage and dendritic cell inform $\mathrm{T}$ cells of the normal, abnormal or foreign trash in the neighborhood that is administered by the draining lymph node. T cells are management but they do not act unless directed by the dendritic cells. Dendritic cells direct whereas $\mathrm{T}$ cells tell other cells what to do through cell-cell interactions and with specific cytokine comments. The nature of the local and systemic response is determined by the nature of the cytokine conversation. The normal status quo environment is maintained by interleukin (IL10) and transforming growth factor beta (TGFbeta) produced by regulatory $\mathrm{T}$ cells and other cells. These cytokines will also promote a return to the status quo and facilitate memory of a response after a challenge. IL4, IL 5 and IL10 in a Th2 response facilitate trash removal by promoting antibody production which includes potent opsonins. IL17, IL23 and tumor necrosis factor alpha (TNFalpha) promote a pro-inflammatory, neutrophil driven Th17 response while IL2, interferon gamma and tumor necrosis factor beta (TNFbeta) define a more complete and long lasting pro-inflammatory, cell-mediated and antibody response to the challenge.

Looking at antibodies as very specific opsonins that facilitate trash pick-up provides a different perspective on their role in immune protections. For normal trash, there are natural IgM, IgG3 and IgA antibodies. ${ }^{3}$ These are antibodies produced primarily by the B1 type of $\mathrm{B}$ cell. The antibodies facilitate opsonization by providing low level, less specific binding to a broad array of molecules including self proteins. Rheumatoid factor, a unique type of natural IgM antibody, binds to antigen-antibody complexes and can facilitate opsonization by increasing the avidity of the interaction. The presence of a large 
amount of protein in the body triggers an antibody response from classical B2 B cells to facilitate its removal from the body. This would be managed by a Th2 directed helper $\mathrm{T}$ cell response and would include a more sophisticated (class switch to $\operatorname{IgG}$, $\operatorname{IgE}$ or $\operatorname{IgA}$ ), stronger antibody response (affinity maturation) than the natural antibody response and also immune memory. Most vaccines consist of a bolus of antigen, protein or carbohydrate, and this will elicit a Th2 antibody response to facilitate its subsequent clean-up. As such, Th2 responses are part of the status quo, garbage clean-up type of response. A large component of the trash pickup for macrophages consists of apoptotic cells. This process is termed efferocytosis. ${ }^{4}$ Apoptotic cells expose many "eat me" signals but also expose immunosuppressive signals. Upon apoptosis, the membrane structure changes, blebs form and phospholipid serine ${ }^{5}$ is externalized to become both an 'eat me' and a 'don't get excited' immunosuppressive signal ${ }^{6,7}$ This allows the macrophages to clean up the constant generation of apoptotic cells without promoting autoimmune responses. Efferocytosis of neutrophils also provides a signal to macrophages to switch from inflammation to repair to promote wound healing.

During infection, microbes and their components are also molecular trash. Unlike normal body trash, microbial trash is decorated with toxic, sharp or similarly dangerous molecules which express pathogen associated molecular patterns (PAMP). These molecules make macrophage and dendritic cells angry and reactive. Th17 (CD4), Th1 (CD4) and Tc1 (CD8) responses are induced and these further provoke macrophages and dendritic cells with cytokines to get them angry. These are the reactions that usually interest and excite immunologists. PAMPS are recognized by Toll-like receptors and other PAMP receptors (PAMPR). For bacteria, PAMPS include cell wall components such as lipopolysaccharide, lipoteichoic acids, and peptidoglycan. Similarly, for fungi, they are the cell wall components. For viruses, PAMPs include viral RNA and non-nuclear DNA. Upon host cell disruption, damage-associated molecular patterns (DAMPs) are released that in excess can also aggravate macrophage and dendritic cells. These molecules include extracellular matrix components, non-nuclear DNA or released RNA, nucleosides and nucleotides such as AMP, ADP or ATP, or secreted proteins such as heat shock proteins or beta defensins. These molecules are sensed by specific DAMP receptors (DAMPR). Binding of DAMPs or PAMPs to their receptors trigger activation cascades that promote the release of proinflammatory cytokines and chemokines and for dendritic cells, their maturation and movement to the lymph node.

Surrounding the site of infection, an inflammatory war zone is created. DAMPS and PAMPs trigger responses that enhance blood flow and leakage of complement and other molecules into the infected site and with activation of complement, the recruitment of neutrophils and monocytes. The $\mathrm{C} 3 \mathrm{~b}$ component of complement also acts as an opsonin and later, the $\mathrm{C} 3 \mathrm{~d}$ component promotes antibody production by B cells to facilitate trash clearance. Dendritic cells at the site pick up garbage that contains PAMPS or DAMPS. They respond to the stimulus, spread a local cytokine alarm, mature, and then run to the lymph node to direct $\mathrm{T}$ cells to act. During their maturation and trip to the lymph node, the dendritic cells cease to be garbage pickers, but continue to process the protein trash that they have acquired and then display their peptide pickings on MHC I and MHC II molecules on the cell surface. During maturation, they also make and express activator molecules on their surface. With their cytokines and cell surface proteins, the dendritic cells activate and determine the response for those $\mathrm{T}$ cells that can recognize the displayed peptides representative of the microbe at the site of the infection. The cytokines produced by the Th17 and Th1 T cells authorize and sanction the defensive and combative proinflammatory nature of immune and other cells. As the Th17 and Th1 cells mature and grow in number, they leave the lymph node to spread the alarm and support neutrophil and macrophage functions, respectively. Mature CD4 T cells wander into and through the skin and other tissues. The Th17 cells reinforce the neutrophil response. A little later and for more permanence, a Th1 response occurs and with their cytokines (interferon gamma and tumor necrosis factor alpha) reinforces the angry state. When the CD4 Th1 cells wander into an infected area and meet a macrophage that displays their peptide on a MHC II molecule, they bind and release interferon gamma. This activates the antimicrobial weapons of the irate macrophage making it angrier and promoting the killing of whatever it has phagocytized. These angry macrophages release more inflammatory cytokines which enhance the inflammation. The Th1 T cells also promote the class switch and production of immunoglobulin $\mathrm{G}$ ( $\mathrm{IgG}$ ) by B cells.

Eventually, the large amount of debris produced by the battle will be delivered in lymph to lymph nodes and trigger Th2 responses. The Th2 response will promote expansion and maturation of the antibody response to promote clearance of the antigen. Later, Treg responses will be activated to return the system to status quo. This type of response invokes out all the elements of the immune response and incurs a very vivid and long lasting memory. Live vaccines and vaccines with adjuvants that include PAMPs or DAMPS mimic the response to an infection. Instead of eliciting just a Th2 related antibody response to clean up a bolus of protein debris, a complete $\mathrm{T}$ and $\mathrm{B}$ cell response with better memory responses is generated. Sometimes the system goes awry and autoimmunity results. The production of a large amount of cytokine (cytokine storm) during a bacterial or viral infection can expand the inflammatory zone beyond the local site to the rest of the body to activate and anger macrophages and dendritic cells. Instead of performing the status quo clean up of normal cellular or tissue debris, these signals override normal regulatory signals so that host peptides on MHC I and MHC II molecules from the debris are presented to $\mathrm{T}$ cells with the same infection warning as for the microbe. This gives the $\mathrm{T}$ cells and subsequently the B cells permission to activate cytolytic and inflammatory immune responses against cells expressing these self proteins to create an autoimmune response ${ }^{8,9}$ Fortunately, this does not happen to everybody, only to those people who have the MHC I of MHC II molecules that can bind and display peptides from certain normal tissue proteins.

This garbage analogy helps explain much of the immune response and can be extended to include other aspects. Specifically, it helps explain why there are natural autoantibodies, why deficiencies in complement components can result in autoimmunity and helps put immuno tolerance and autoimmunity into perspective. However, as with other analogies, it has its limitations. I leave it up to the reader to see the limitations and create extensions as a means to better understand the immune response.

\section{Acknowledgments}

I want to thank my students for not discouraging my use of analogies in teaching.

\section{Conflicts of interest}

The author has no conflicts of interest.

\section{Funding}

None. 


\section{References}

1. Rashed MH, Bayraktar E, Helal GK, A et al. Exosomes: From Garbage Bins to Promising Therapeutic Targets. Int J Mol Sci. 2017;18(3):538

2. Truedsson L, Bengtsson AA, Sturfelt G Complement deficiencies and systemic lupus erythematosus. Autoimmunity. 2009;40(8):560-566.

3. Gronwall C, Silverman GJ Natural IgM:Beneficial autoantibodies for control of inflammatory and disease? J Clin Immunol. 2014;34(1):S12S21.

4. Munoz LE, Herrman M, Berens C. Dying autologous cells as instructors of the immune system. Clinical and Experimental Immunology. 2014;179(1):1-4

5. Birge RB, Boeltz S, Jumar S, et al. Phosphatidylserine is a global immunosuppressive signal in efferocytosis, infectious disease and cancer. Cell Death Differ. 2016;23(6):962-978.
6. Elliott MR, Koster KM, Murphy PS Efferocytosis Signaling in the Regulation of Macrophage Inflammatory Responses. J Immunol. 2017;198(4):1387-1394

7. Nagata S Hanayama R, Kawane K Autoimmunity and the Clearance of Dead Cells. Cell. 2010;140(5):619-630.

8. Getts DR, Chastain EML, Terry RL, et al. Virus infection, antiviral immunity, and autoimmunity. Immunological Reviews. 2013;255(1):197-209.

9. Franceschi C, Garagnani P, Vitale, et al. Inflammaging and 'Garbaging'. Trends in Endocrinology \& Metabolism. 2017;28(3):199-212. 